\section{Public Attitudes to Science}

The Commission of the European Communities has recently published the results of a programme of research into the attitudes adopted by the public in the member countries towards science; some of the principal findings are summarized below. They suggest that the commonly expressed view of a general disenchantment is unduly pessimistic.

An opinion poll was conducted in April-May 1977 on a representative sample of the adult populations of the nine countries of the European Communities. Altogether, 9000 people were questioned personally in their homes by qualified interviewers who used a questionnaire carrying about twenty questions strictly standardized for all countries.

The questionnaire covered the following aspects :

1. How is science regarded in general ? Is it seen as beneficial or not? Is its present image largely conditioned by past achievements and setbacks? Does it offer a hopeful future? 2. If people believe in a future for science, does that future deserve to be subsidized?

3. On what scale - national or European - should scientific research efforts be subsidized?

4. Are there any priority tasks to be assigned to scientific research? What are they? On the other hand, are there any tasks which should be "urgently" relegated to the "waiting list" ? 5. Are people interested in scientific information put out via the mass media? What kind of information? What, in particular, is the impact of presentday scientists who appear on television?

\section{Results of the Poll}

Science is widely regarded by the public as one of the principal means of improving its existence: This definition was chosen by $69 \%$ from a list of five proposed (three of which were unfavourable).

To the question: which of the following opinions corresponds best to what you think about science, the answers were :

$14 \%$ : All it does is to satisfy the curiosity of scientists, or it produces more disadvantages than advantages, or it is dangerous. $6 \%$ : It is exciting. $69 \%$ : It is one of the most important factors in the improvement of our daily life.

$11 \%$ : No opinion.

However, this confidence is tinged with apprehension because $67 \%$ also believe that very dangerous repercussions can ensue from civilian applications of science.

That apprehension is not sufficient to make people doubt the importance of science. Not only do they recognize the important role of science in the positive development of living conditions in the past 25 years, but the large majority believes in the future of scientific research including research to improve life in the developing countries $(80 \%)$.

To the question : all things considered, do you think there are still good or beneficial things left to discover in science, $89 \%$ replied in the affirmative.

It is regarded as self-evident that the State should subsidize scientific research $(81 \%)$. More unexpectedly, it was regarded as equally self-evident that the research should be pursued by a pooling of effort between the Member States of the Communities $(79 \%)$ rather than in each country separately.

This was in response to the question : as scientific research is extremely expensive, even though it can bring advantages and prestige to the country where it goes on, in your opinion, would you prefer that :

European States should get together to pool their scientific research?

Each country should do its research for itself?

Of the various scientific disciplines mentioned in the questionnaire, some came in for considerable attention while others received hardly any. The priority importance of medical research in the eyes of the general public is not surprising. It stems not only from the fact that the public spontaneously realizes the personal benefit it can expect from such research, but also from the fact that this scientific field is by far the most publicized by

Agricultural research to meet the world's food requirements comes in second place, and research to combat and control pollution comes third.

Other priority fields are the search for new forms of energy and the prevention and treatment of drug-taking. sixth.

On the other hand, the public is willing to forego, or at least reduce, space exploration and research on weapons and national defence.

Research in the fields of meteorology the media, especially television.

Safety of nuclear installations comes and climate control at present receives little attention; it appears to be little known by the public.

Finally, the great confidence of the public in science is accompanied by an equally great interest in scientific information. This is borne out by people's attention to, and interest in, news of scientific activities, the frequency of private discussions about such news, the very positive impact of television broadcasts involving the participation of scientists, and the fact that only a small proportion of people did not answer the questions in the survey.

These various results are surprising on more than one count. But the main surprise - and this must be stressed - no doubt lies in the extremely strong and widespread consensus in favour of science. The various analyses, which are set out in detail in the survey report, provide facts and figures that confirm the strength of this consensus :

It is also interesting that:

- an extremely high proportion of those interviewed gave identical or almost identical replies to the ten main questions ;

- the replies were remarkably standard from one country to another ;

- the consensus was independent of the variables which are usually found to influence opinions, such as age, level of education, political views, socio-economic category, and even social position and scales of values ; - systematic analysis of the few patterns of opinion divergent from the consensus, the number of which never exceeded $6 \%$ of the whole, failed to reveal any hard core of hostility to science.

In conclusion, it may be said that the survey provides clear evidence that there is no crisis of confidence in science among the general public. Of course, the question is not thereby closed. The ambivalence of the apprehensive confidence in scientific research suggests that some other lines of inquiry should be explored: for instance; What is the public's confidence in this or that particular field? What thresholds of risk are accepted by the public, i.e., those beyond which its confidence is withdrawn? What types of information are best suited to fostering the latent interest, if not the enthusiasm for science? And, on the other hand, what types of information are most likely to shake their confidence by nurturing anxiety? There is no doubt that the circulation of this survey in interested quarters will prompt more suggestions for new research, inspired by the strong consensus that is revealed. 